\title{
Flux Front Penetration in Disordered Superconductors
}

\author{
Stefano Zapperi, ${ }^{1}$ André A. Moreira, ${ }^{2}$ and José S. Andrade, $\mathrm{Jr}^{2}$ \\ ${ }^{1}$ INFM sezione di Roma 1, Dipartimento di Fisica, Università "La Sapienza," P.le A. Moro 2, 00185 Roma, Italy \\ ${ }^{2}$ Departamento de Física, Universidade Federal do Ceará, 60451-970 Fortaleza, Ceará, Brazil
}

(Received 7 November 2000)

\begin{abstract}
We investigate flux front penetration in a disordered type-II superconductor by molecular dynamics simulations of interacting vortices and find scaling laws for the front position and the density profile. The scaling can be understood by performing a coarse graining of the system and writing a disordered nonlinear diffusion equation. Integrating numerically the equation, we observe a crossover from flat to fractal front penetration as the system parameters are varied. The value of the fractal dimension indicates that the invasion process is described by gradient percolation.
\end{abstract}

DOI: $10.1103 /$ PhysRevLett.86.3622

The magnetization properties of type-II superconductors have been studied for many years, but the interest in this problem has been renewed with the discovery of high temperature superconductors [1]. The magnetization process is usually described in terms of the Bean model [2] and its generalizations: flux lines enter into the sample and, due to the presence of quenched disorder, give rise to a steady flux gradient. While the Bean model provides a consistent picture of average magnetization properties, such as the hysteresis loop and thermal relaxation effects [3], it does not account for local fluctuations in time and space. It has been recently observed that flux line dynamics is intermittent, taking place in avalanches [4], and flux fronts are not smooth [5-7]. In particular, it has been shown that the flux front crosses over from flat to fractal as a function of material parameters and applied field [6].

A widely used modeling strategy to describe the fluctuations around the Bean state consists of molecular dynamics (MD) simulations of interacting flux lines, pinned by quenched random impurities [8-13]. With this approach it has been possible to reproduce flux profiles [10], hysteresis [10], avalanches [9,12,13], and plastic flow $[8,13]$. One of the aims of these studies [10] is to establish precise connections between the microscopic models and the macroscopic behavior, captured, for instance, by generalized Bean models. A different approach treats the problem at mesoscopic scale, describing the evolution of interacting coarse-grained units $[14,15]$, supposed to represent the system at an intermediate scale. While these models give a faithful representation of several features of the problem, the connection with the underlying microscopic dynamics remains unexplored.

In this Letter, we investigate the invasion of magnetic flux into a disordered superconductor, a problem that has recently been the object of intense experimental research [5-7]. We first analyze the problem by MD simulations, in analogy with Ref. [10], and obtain scaling laws relating the front position and the flux profile to the pinning strength. In order to understand these results, we perform a coarse graining of the equation of motion and obtain a nonlinear diffusion equation for the flux line density. In the absence
PACS numbers: 74.60.Ge, 05.45.-a, 47.55.Mh

of pinning, the equation reduces to the model discussed in Refs. [16,17]. This model has been analytically $[16,17]$ solved to provide expressions for the initial dynamics of the front that are in agreement with our MD simulations. We show that when quenched disorder is included in the diffusion equation, the flux front roughens and is eventually pinned. Varying the parameters of the model (applied field, disorder, interaction strength), the fluctuations of the front display a crossover from flat to fractal that is consistent with experimental observations [5-7]. The value of the fractal dimension suggests that the strong disorder limit is described by percolation.

In an infinitely long cylinder, flux lines can be modeled as a set of interacting particles performing an overdamped motion in a random pinning landscape [8-13]. The equation of motion for each flux line $i$ can be written as

$$
\Gamma \vec{v}_{i}=\sum_{j} \vec{J}\left(\vec{r}_{i}-\vec{r}_{j}\right)+\sum_{p} \vec{G}\left[\left(\vec{R}_{p}-\vec{r}_{i}\right) / l\right]+\eta\left(\vec{r}_{i}, t\right),
$$

where the effective viscosity can be expressed in terms of material parameters $\Gamma=\Phi_{0} H_{c 2} / \rho_{n} c^{2}$. Here, $\Phi_{0}$ is the magnetic quantum flux, $c$ is the speed of light, $\rho_{n}$ is the resistivity of the normal phase, and $H_{c 2}$ is the upper critical field. The first term on the right-hand side represents the vortex-vortex interaction and it is given by $\vec{J}(\vec{r}) \equiv$ $\left[\Phi_{0}^{2} /\left(8 \pi \lambda^{3}\right)\right] K_{1}(|\vec{r}| / \lambda) \hat{r}$, where the function $K_{1}$ is a Bessel function decaying exponentially for $|\vec{r}|>\lambda$ and $\lambda$ is the London penetration length [18]. The second term on the right-hand side accounts for the interaction between pinning centers, modeled as localized traps, and flux lines. Here, $\vec{G}$ is the force due to a pinning center located at $\vec{R}_{p}$, $l$ is the range of the wells (typically $l \ll \lambda$ ), and $p=$ $1, \ldots, N_{p}$ ( $N_{p}$ is the total number of pinning centers). While Refs. [9-11] employed parabolic traps, we decided to avoid discontinuities in the force and used instead $\vec{G}(\vec{x})=-f_{0} \vec{x}(|\vec{x}|-1)^{2}$, for $|\vec{x}|<1$ and zero otherwise. Finally, we add to the model an uncorrelated thermal noise term $\eta$, with zero mean and variance $\left\langle\eta^{2}\right\rangle=k_{b} T / \Gamma$. Although in the present simulations we restrict ourselves to 
the case $T=0$ (see Ref. [19] for the implementation of thermal noise in MD simulations), we will discuss the effect of temperature in the coarse-grained description of the dynamics.

We perform MD simulations based on Eq. (1) and analyze the flux front propagation for different values of the pinning strength $f_{0}$. We use $N=5000$ flux lines in a system of size $\left(L_{x}=800 \lambda, L_{y}=100 \lambda\right.$ [20]), with $N_{p}=$ 800000 Poisson distributed pinning centers of width $l=$ $\lambda / 2$, corresponding to a density of $n_{0}=10 / \lambda^{2}$. The injection of magnetic flux into the sample is implemented similarly to Ref. [10], concentrating at the beginning of the simulation all the flux lines in a small strip, parallel to the $y$ direction, of length $L^{\prime}=10^{-2} \lambda$ and imposing periodic boundary conditions in both directions. The front position $x_{p}$ is identified as the $x$ coordinate of the most advanced particle in the system at different times. In Fig. 1 we show that $x_{p}$ grows initially with time as $t^{1 / 3}$ and eventually saturates to a value $\xi_{p}$ which increases as the strength of the pinning centers $f_{0}$ is decreased. The data collapse shown in the inset of Fig. 1 indicates that the front pinning length $\xi_{p}$ scales as $f_{0}^{1 / 2}$. When the front saturates, we measure the density profiles [10] which can be collapsed using the scaling form $\rho(x)=f_{0}^{-1 / 2} F\left(x f_{0}^{1 / 2}\right)$ (see Fig. 2). In addition, we vary the density of pinning centers, using $n_{0} \lambda^{2}=2.5,5,7.5$, and 10 , and find that similar scaling collapses hold if $f_{0}$ is replaced by $f_{0} \sqrt{n_{0}}$.

In order to understand these results, we perform a coarse graining of Eq. (1), starting from the Fokker-Plank equation for the probability distribution of the flux line coordi-

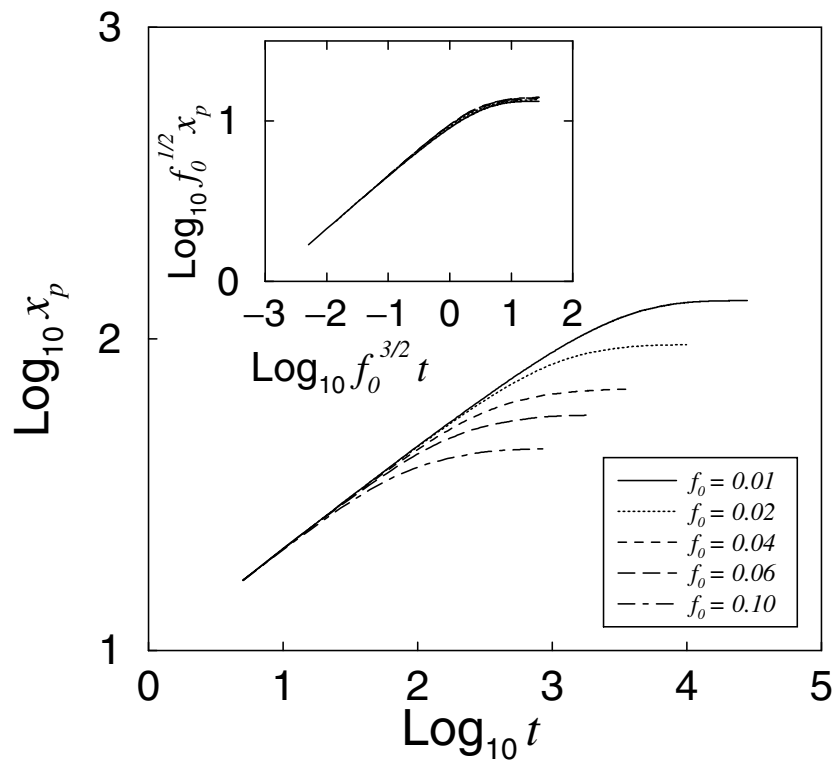

FIG. 1. The average position of the front, obtained from MD simulations injecting a constant magnetic flux from the boundary, is plotted as a function of time. The curve increases as $t^{1 / 3}$ and saturates at long times to a value depending on $f_{0}$. In the inset, we show by data collapse that the pinning length scales as $f_{0}^{-1 / 2}$. nates $P\left(\vec{r}_{1}, \ldots, \vec{r}_{N}, t\right)$,

$$
\Gamma \frac{\partial P}{\partial t}=\sum_{i} \vec{\nabla}_{i}\left(-\vec{f}_{i} P+k_{B} T \vec{\nabla}_{i} P\right),
$$

where $\vec{f}_{i}$ is the force on the particle $i$ given by Eq. (1). Next, we introduce the single particle density $\rho(\vec{r}, t) \equiv$ $\left\langle\sum_{i} \delta^{2}\left(\vec{r}-\vec{r}_{i}\right)\right\rangle$, where the average is done over the distribution $P\left(\vec{r}_{1}, \ldots, \vec{r}_{N}, t\right)$. The evolution of $\rho$ can be directly obtained from Eq. (2) and is given by

$$
\begin{aligned}
\Gamma \frac{\partial \rho}{\partial t}=-\vec{\nabla}\left\{\int d^{2} r^{\prime} \vec{J}\left(\vec{r}-\vec{r}^{\prime}\right) \rho^{(2)}\left(\vec{r}, \vec{r}^{\prime}, t\right)\right. \\
\left.-\sum_{p} \vec{G}\left[\left(\vec{R}_{p}-\vec{r}\right) / l\right] \rho(\vec{r}, t)\right\}+k_{B} T \nabla^{2} \rho,
\end{aligned}
$$

where $\rho^{(2)}\left(\vec{r}, \vec{r}^{\prime}, t\right)$ is the two-point density, whose evolution depends on the three-point density, and so on. The simplest truncation scheme involves the approximation $\rho^{(2)}\left(\vec{r}, \vec{r}^{\prime}, t\right) \simeq \rho(\vec{r}, t) \rho\left(\vec{r}^{\prime}, t\right)$. We then coarse grain the equation considering length scales larger than $\lambda$. This can be done by expanding $\vec{J}$ in Fourier space, keeping only the lowest order term in $\vec{q}$, and retransforming back in real space. The result reads

$$
\int d^{2} r^{\prime} \vec{J}\left(\vec{r}-\vec{r}^{\prime}\right) \rho\left(\vec{r}^{\prime}, t\right) \simeq-a \vec{\nabla} \rho(\vec{r}, t),
$$

where $a \equiv \int d^{2} r \vec{r} \cdot \vec{J}(\vec{r}) / 2=\Phi_{0}^{2} / 4$.

The coarse graining of the disorder term is more subtle. A straightforward elimination of short wavelength modes would give rise, as in the previous case, to a random force $\vec{F}_{c}(\vec{r})=-g \vec{\nabla} n$, where $n$ is the coarse-grained version of the microscopic density of pinning centers $\hat{n}(\vec{r}) \equiv \sum_{p} \times$ $\delta^{2}\left(\vec{r}-\vec{R}_{p}\right)$ and $g \propto f_{0}$. This method cannot be applied

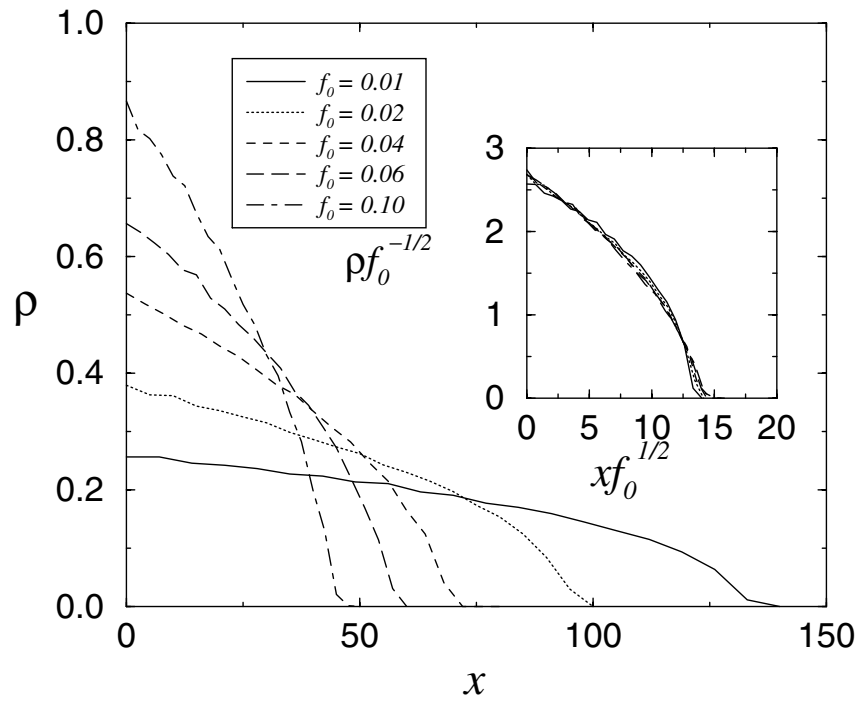

FIG. 2. The pinned density profiles measured in MD simulations. In the inset we show the data collapse. 
for short-range attractive pinning forces such as the one we are investigating. In this case, short wavelength modes yield a macroscopic contribution to pinning that cannot be neglected. Consider, for instance, the flow between two coarse-grained regions: short-range microscopic pinning forces give rise to a macroscopic force that should always oppose the motion, while the random force derived above could, in principle, point in the direction of the flow. In other words, $F_{c}(\vec{r})$ should be considered as a friction force [21] whose direction is always opposed to the driving force $\vec{F}_{d}$ (in our case $\vec{F}_{d}=a \vec{\nabla} \rho$ ) and whose absolute value is given by $|g \vec{\nabla} n|$ for $\left|\vec{F}_{d}\right|>|g \vec{\nabla} n|$ and to $\left|\vec{F}_{d}\right|$ otherwise [22].

Collecting all the terms, we finally obtain a disordered nonlinear diffusion equation for the density of flux lines

$$
\Gamma \frac{\partial \rho}{\partial t}=\vec{\nabla}\left(a \rho \vec{\nabla} \rho-\rho \vec{F}_{c}\right)+k_{B} T \nabla^{2} \rho .
$$

The boundary conditions representing our MD simulations correspond to a constant number of flux lines $M L_{y}$ injected into the system, so that the total density is conserved $\int d x d y \rho(x, y, t)=M L_{y}$. With these boundary conditions, the $T=0$ behavior depends on a single effective coupling constant $g_{0} \equiv g \sqrt{n_{0}} /(a M)$ as can be shown by rescaling Eq. (5) and the conservation law as

$$
\rho=\tilde{\rho} / M, \quad t=\tilde{t} /\left(\Gamma a M^{2}\right), \quad n=\tilde{n} \sqrt{n_{0}} .
$$

In the limit $g_{0}=0$ and $T=0$, Eq. (5) was solved by Bryskin and Dorogotsev [16] using similarity methods, and the solution reads $\rho(x, y, t)=t^{-1 / 3} h\left(x / t^{1 / 3}\right)$, where $h(u)=\left(1-u^{2}\right) / 6$ for $u<1$ and vanishes for $u \geq 1$ $[16,17,23]$. Notice that we recover here the $t^{1 / 3}$ behavior observed in MD at early times for the front position [24]. When disorder is present $\left(g_{0}>0\right)$, we expect the front to deform and eventually encounter a strong pinning region where it stops. The scaling of the front position $\xi_{p}$ with $f_{0}$ observed in MD simulations can be explained, noticing that the front will be pinned when the force due to the density gradient is equal to the friction force of the order of $f_{0} \sqrt{n_{0}}$. The gradient force can be estimated as $a \nabla \rho \sim$ $\rho_{0} / \xi_{p}$, where the density at the boundary is given by $\rho_{0} \sim$ $M / \xi_{p}$. Thus we find that $\xi_{p} \sim\left(M a / f_{0} \sqrt{n_{0}}\right)^{1 / 2}=g_{0}^{-1 / 2}$, in agreement with MD simulations.

In order to confirm the validity of these considerations, we discretize Eq. (5) on a tilted square lattice and integrate the equations numerically by using a finite volume technique with an upwind scheme to avoid numerical instabilities. At the beginning of the simulation, all flux lines are concentrated at the boundary of the sample (i.e., $x=0$ ) and in each site of the lattice we define an uncorrelated Gaussian pinning center density $n$, with mean $n_{0}$ and variance $\sqrt{n_{0}}$, thus defining a local random friction force. As flux lines enter into the sample, we identify the flux front by using a burning algorithm and compute its average position $x_{p}$. In the initial stage, $x_{p}$ grows as $t^{1 / 3}$ up to a crossover length scaling as $\xi_{p} \sim g_{0}^{-1 / 2}$, in agreement with MD simulations (see Fig. 3). In addition, we measure the density profiles and find that they rescale with $g_{0}$ in the same way as in MD simulations.

The numerical integration of the diffusion equation allows for a direct analysis of the fluctuations in the front as a function of different internal parameters. Measuring the width $W$ of the fronts as a function of time for different values of $g_{0}$, we find that in the initial stage $W$ grows as a power law $t^{\beta}$ where $\beta \simeq 0.35$ until it saturates to a value that decreases with $g_{0}$. Thus the front crosses over from flat to fractal as it enters into the material. In principle, we can control the strength of the fluctuations and the associated characteristic length $\xi^{*}$ by tuning $g_{0}$, which directly reflects experimentally measurable parameters.

In order to compare the model with experiments, we have to implement appropriate boundary conditions. In Refs. $[5,6]$ the external field was ramped at a constant rate, which corresponds to a constant increase of the boundary density: $\rho(0, y, t)=h t$ [17]. Integrating the equation with this boundary condition, we observe that for strong disorder the flux front roughens, displaying substantial overhangs (see the inset of Fig. 4 [25]). Here the box counting method is applied to estimate the fractal dimension of the front: we divide the lattice into boxes of size $b$ and count the number of occupied boxes $N(b)$, which decays as $b^{-D_{f}}$, where $D_{f}$ is the fractal dimension. Figure 4 shows that for $b<\xi^{*}$ the data are well fitted by a power law with exponent $D_{f}=4 / 3$, which coincides with the fractal dimension of the perimeter of the percolation cluster. For $b>\xi^{*}$ the fractal dimension crosses over to $D_{f}=1$, corresponding to a flat front. The observed behavior is reminiscent of gradient percolation [26], a model in which the concentration of occupied sites $p$ decreases with the distance from the sample boundary. The invaded area is

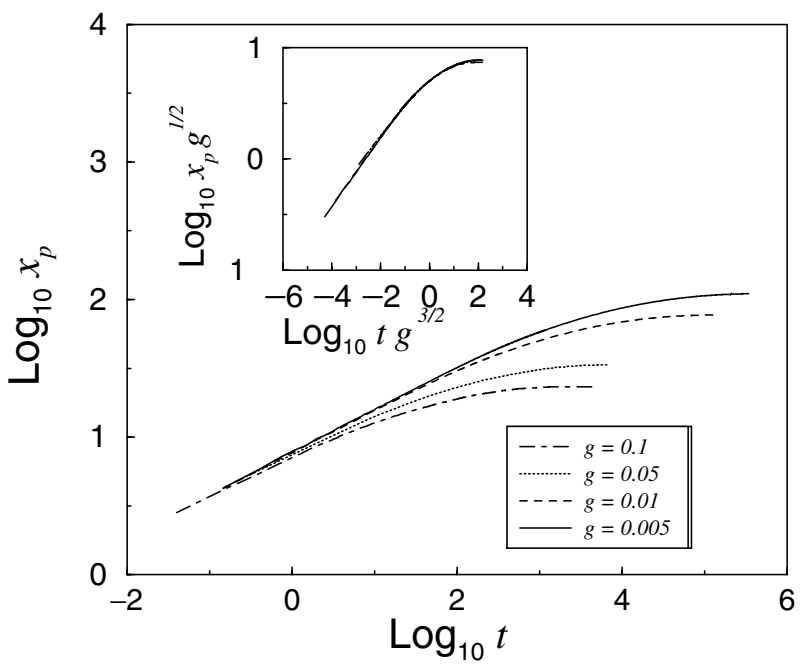

FIG. 3. The front position measured by simulating the continuum equation for different values of $g$. In the inset we show the data collapse. 


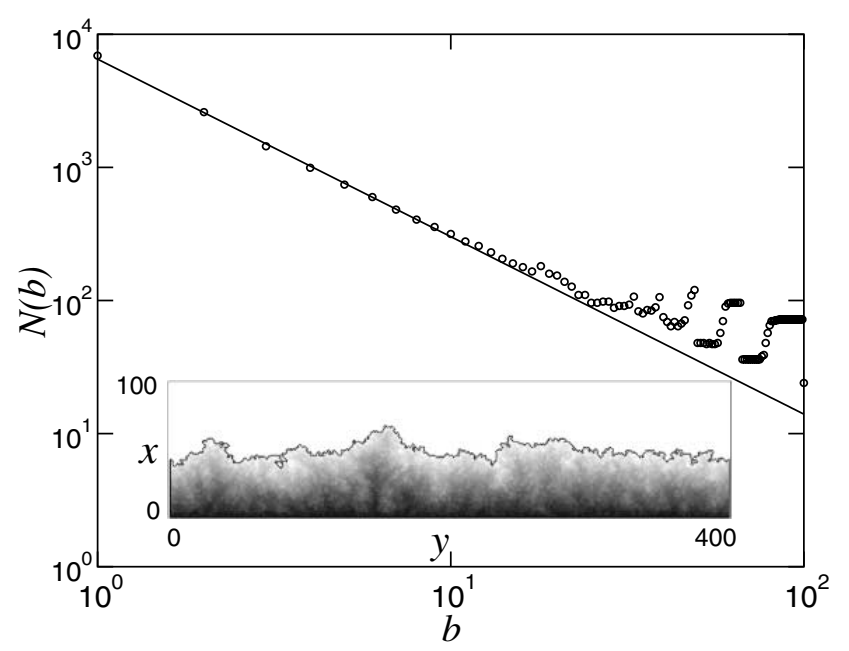

FIG. 4. In the inset we show a density plot obtained by simulating the continuum equation with $g=5, a=6.28, n_{0}=8$, and concentration at the boundary increasing at rate $h=0.01$. In the main figure we report the box counting plot of the front, averaged over 12 realizations of the disorder. The front has fractal dimension $D_{f}=4 / 3$ (solid line) and crosses over to $D_{f}=1$ at large length scales.

compact, but its perimeter is fractal (with $D_{f}=4 / 3$ ) up to a length scale $\xi^{*}$ which depends on the concentration gradient.

In experiments on flux penetration in $\mathrm{Tl}_{2} \mathrm{Ba}_{2} \mathrm{CuO}_{6+x}$ thin films with controlled anisotropy, $D_{f}$ was found to change continuously from $D_{f} \simeq 1.5$ to $D_{f} \simeq 1$ as the degree of anisotropy and the applied field was varied [6]. It would be interesting to check if the observed continuous variation of the fractal dimension can be explained by a crossover from a percolation process at short length scales to a flat invasion at large length scales, with a crossover length depending, for instance, on the degree of anisotropy.

In conclusion, we have analyzed flux front penetration in disordered type-II superconductors, establishing connections between microscopic flux line dynamics and a mesoscopic nonlinear diffusion equation. This equation allows one to interpret the results of MD simulations and provides a useful framework to analyze experiments.

This work was supported by CNPq and FUNCAP. We thank A. Baldassarri, K. Behnia, M. C. Miguel, S. Sarti, A. Scala, A. Vespignani, and R. J. Wijngaarden for useful discussions. S.Z. wishes to thank the Physics Department of UFC, where this work was initiated, for its hospitality.

[1] For extensive reviews, see E. H. Brandt, Rep. Prog. Phys. 58, 1465 (1995); G. Blatter, M. V. Feigel'man, V. B. Geshkenbein, A. I. Larkin, and V. M. Vinokur, Rev. Mod. Phys. 66, 1125 (1994).

[2] C. P. Bean, Rev. Mod. Phys. 36, 31 (1964).
[3] Y. B. Kim, C. F. Hempstead, and A. R. Strnad, Phys. Rev. 129, 528 (1963).

[4] S. Field, J. Witt, F. Nori, and X. Ling, Phys. Rev. Lett. 74, 1206 (1995); C. M. Argenter, Phys. Rev. E 58, 1438 (1998); K. Behnia, C. Capan, D. Mailly, and B. Etienne, Phys. Rev. B 61, R3815 (2000).

[5] R. Surdeanu, R. J. Wijngaarden, E. Visser, J. M. Huijbregtse, J. H. Rector, B. Dam, and R. Griessen, Phys. Rev. Lett. 83, 2054 (1999).

[6] R. Surdeanu, R. J. Wijngaarden, B. Dam, J. Rector, R. Griessen, C. Rossel, Z.F. Ren, and J. H. Wang, Phys. Rev. B 58, 12467 (1998).

[7] S. S. James, S. B. Field, J. Seigel, and H. Shtrikman, Physica (Amsterdam) 332C, 445 (2000).

[8] H. J. Jensen, A. Brass, and A. J. Berlinsky, Phys. Rev. Lett. 60, 1676 (1988).

[9] O. Pla and F. Nori, Phys. Rev. Lett. 67, 919 (1991); R. Richardson, O. Pla, and F. Nori, Phys. Rev. Lett. 72, 1268 (1994); W. Barford, W. H. Beere, and M. Steer, J. Phys. Condens. Matter 5, L333 (1993).

[10] C. Reichhardt, C. J. Olson, J. Groth, S. Field, and F. Nori, Phys. Rev. B 52, 10441 (1995); 53, R8898 (1996).

[11] C. Reichhardt, J. Groth, C. J. Olson, S. Field, and F. Nori, Phys. Rev. B 54, 16108 (1996); 56, 14196 (1997).

[12] O. Pla, N. K. Wilkin, and H. J. Jensen, Europhys. Lett. 33, 297 (1996).

[13] C. J. Olson, C. Reichhardt, and F. Nori, Phys. Rev. B 56, 6175 (1997); Phys. Rev. Lett. 80, 2197 (1998).

[14] W. Barford, Phys. Rev. B 56, 425 (1997).

[15] K.E. Bassler and M. Paczuski, Phys. Rev. Lett. 81, 3761 (1998); G. Mohler and D. Stroud, Phys. Rev. B 60, 9738 (1999); R. Cruz, R. Mulet, and E. Altshuler, Phys. Rev. B 63, 094501 (2001).

[16] V. V. Bryskin and S. N. Dorogotsev, JEPT 77, 791 (1993); Physica (Amsterdam) 215C, 173 (1993).

[17] J. Gilchrist and C. J. van der Beek, Physica (Amsterdam) 231C, 147 (1994).

[18] P.-G. de Gennes, Superconductivity of Metals and Alloys (Benjamin, New York, 1966).

[19] D. Monier and L. Fruchter, Eur. Phys. J. B 17, 201 (2000).

[20] The results do not depend on $L_{y}$ if the flux line density is held constant.

[21] The lattice model described in Ref. [15] implements a random force rather than a friction force, which is used instead in Ref. [14]. We have checked that a random force produces a different scaling for the front position and the profiles than MD simulations.

[22] For a derivation of a friction force from elastic interactions with pinning centers, see C. Caroli and P. Nozieres, Eur. Phys. J. B 4, 23 (1998).

[23] This functional form holds only in the initial regime, when the effect of disorder is weak.

[24] In the continuum equation, the scaling exponent depends on the boundary conditions $[16,17]$. We have checked that this feature is also true for MD simulations.

[25] Reference [5] observed self-affine scaling for the flux front. While we cannot exclude that for some parameter range self-affine scaling could apply, this is not the case for the present data.

[26] B. Sapoval, M. Rosso, and J. F. Gouyet, J. Phys. (Paris), Lett. 46, L149 (1985). 